\title{
A Self-assembly Model of Time-Dependent Glue Strength
}

\author{
Sudheer Sahu, Peng Yin, and John H. Reif
}

\begin{abstract}
Self-assembly is a ubiquitous process in which small objects selforganize into larger and complex structures. In 2000, Rothemund and Winfree proposed a Tile Assembly Model as a mathematical model for theoretical studies of self-assembly. We propose a refined self-assembly model in which the glue strength between two juxtaposed tiles is a function of the time they have been in neighboring positions. We then present an implementation of our model using strand displacement reactions on DNA tiles. Under our model, we can demonstrate and study catalysis and self-replication in the tile assembly. We then study the tile complexity for assembling shapes in our model and show that a thin rectangle of size $k \times N$ can be assembled using $O((\log (N)) / \log \log (N))$ types of tiles, demonstrating the glue model has additional capabilities over the prior tiling assembly model. We also describe a method to implement with DNA tiles our model of time-dependant glue strength.
\end{abstract}

\section{Introduction}

\subsection{Motivation}

Self-assembly is a ubiquitous process in which small objects self-organize into larger and complex structures. Examples in nature are numerous: atoms selfassemble into molecules, molecules into cells, cells into tissues, and so on. Recently, self-assembly has also been demonstrated as a powerful technique for constructing nanoscale objects. For example, a wide variety of DNA lattices made from selfassembled branched DNA molecules (DNA tiles) [10, 21, 23-25, 43, 45, 46] have been successfully constructed. Peptide self-assembly provides another nanoscale example [9]. Self-assembly is also used for mesoscale constructions using capillary forces $[8,30]$ or magnetic forces [1].

Sudheer Sahu was a PhD student in Duke University when this research was conducted.

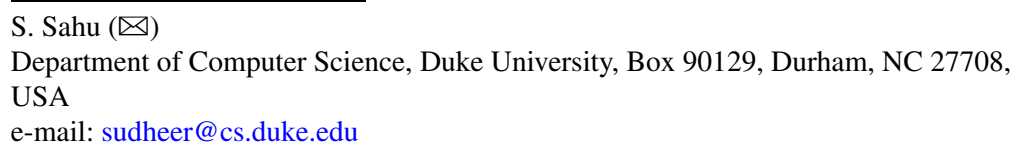




\subsection{Prior Models for Tile Assembly}

Mathematical studies of tiling date back to the 1960s when Wang introduced his tiling model [39]. The initial focus of research in this area was toward the decidability/undecidability of the tiling problem [29]. A revival in the study of tiling was instigated in 1996 when Winfree proposed the simulation of computation [44] using self-assembly of DNA tiles.

In 2000, Rothemund and Winfree [32] proposed an Abstract Tile Assembly (ATA) Model, which is a mathematical model for theoretical studies of self-assembly. This model was later extended by Adleman et al. to include the time complexity of generating specified assemblies [3]. Later work includes combinatorial optimization, complexity problems, fault tolerance, and topology changes, in the abstract tile assembly model as well as in some of its variants [4-7, 11-14, 16, 19, 20, 22, 26, 27 , 31, 33-35, 37, 38, 41, 42].

Adleman introduced a reversible model [2], and studied the kinetics of the reversible linear self-assemblies of tiles. Winfree also proposed a kinetic assembly model to study the kinetics of the self-assembly [40]. Apart from these basic models, various generalized models of self-assembly are also studied [6, 18]: namely, multiple temperature model, flexible glue model, and q-tile model.

\subsection{Needs for New Models for Tile Assembly}

Though all these models contribute greatly toward a good understanding of the process of self-assembly, there are still a few things that could not be easily explained or modeled (for example, the process of catalysis and self-replication in tile assembly). Recall that catalysis is the phenomenon in which an external substance facilitates the reaction of other substances, without itself being used up in the process. A catalyst provides an alternative route of reaction where the activation energy is lower than the original chemical reaction and increase the reaction rate. Adleman [2] has posed an open question if we could model the process of catalysis in the self-assembly of tiles. Self-replication process is one of the fundamental process of nature, in which a system creates copies of itself. For example, DNA is self-replicated during cell division and is transmitted to offspring during reproduction. A material device that can self-replicate is ambition of many engineering disciplines. The biggest incentive is to achieve a low manufacturing cost because self-replication avoids the costs of labor, capital, and distribution in conventional manufactured goods. In an evolving field like nanotechnology, manufacturing costs of molecular machines can become extremely large in the absence of self-replication. Recently, Schulman and Winfree show self-replication using the growth of DNA crystals [36], but their system requires shear forces to separate the replicated units. In this paper, we propose a new model, in which catalysis and selfreplication is possible without external intervention. In our new model, which is 
built on the basic framework of the ATA model, the glue strength between different glues is dependent on the time for which they have remained together.

The rest of the paper is organized as follows. First, we define the prior ATA model as well as our new model formally in Sect. 2.2. We then put forth a method to physically implement such a system in Sect. 3 . Then we present the processes of catalysis and self-replication in tile assembly in our model in Sects. 4 and 5, respectively. In Sect. 6, we discuss the tile complexity of assembly of various shapes in our model, beginning with the assembly of thin rectangles in Sect. 6.1 and the extension to other shapes in Sect. 6.2. We conclude with the discussion of our results and future research directions in Sect. 7.

\section{Tiling Assembly Models}

\subsection{The Abstract Tiling Assembly (ATA) Model}

The Abstract Tile Assembly (ATA) model was proposed by Rothemund and Winfree [32] in 2000. Intuitively speaking, a tile in the ATA model is a unit square where each side of the square has a glue from a set $\Sigma$ associated with it. In this paper, we use the terms pad and side of the tile interchangeably. Formally, a tile is an ordered quadruple $\left(\sigma_{n}, \sigma_{e}, \sigma_{s}, \sigma_{w}\right) \in \Sigma^{4}$, where $\sigma_{n}, \sigma_{e}, \sigma_{s}$, and $\sigma_{w}$ represent the northern, eastern, southern, and western side glues of the tile, respectively. $\Sigma$ also contains a special symbol null, which is a zero-strength glue. $T$ denotes the set of all tiles in the system. A tile cannot be rotated. So, $\left(\sigma_{1}, \sigma_{2}, \sigma_{3}, \sigma_{4}\right) \neq\left(\sigma_{2}, \sigma_{3}, \sigma_{4}, \sigma_{1}\right)$. Also defined are various projection functions $n: T \rightarrow \Sigma, e: T \rightarrow \Sigma, s: T \rightarrow \Sigma$, and $w$ : $T \rightarrow \Sigma$, where $n\left(\sigma_{1}, \sigma_{2}, \sigma_{3}, \sigma_{4}\right)=\sigma_{1}, e\left(\sigma_{1}, \sigma_{2}, \sigma_{3}, \sigma_{4}\right)=\sigma_{2}, s\left(\sigma_{1}, \sigma_{2}, \sigma_{3}, \sigma_{4}\right)=$ $\sigma_{3}$, and $w\left(\sigma_{1}, \sigma_{2}, \sigma_{3}, \sigma_{4}\right)=\sigma_{4}$.

A glue-strength function $g: \Sigma \times \Sigma \rightarrow \mathbb{R}$ determines the glue strength between two abutting tiles. $g\left(\sigma, \sigma^{\prime}\right)=g\left(\sigma^{\prime}, \sigma\right)$ is the strength between two tiles that abut on sides with glues $\sigma$ and $\sigma^{\prime}$. If $\sigma \neq \sigma^{\prime}, g\left(\sigma, \sigma^{\prime}\right)=0$; otherwise, it is a positive value. It is also assumed that $g(\sigma, n u l l)=0, \forall \sigma \in \Sigma$. In the tile set $T$, there is a specified unique seed tile $s$. There is a system parameter to control the assembly known as temperature and denoted as $\tau$. All the ingredients described above constitute a tile system, a quadruple $\langle T, s, g, \tau\rangle$. A configuration is a snapshot of the assembly. More formally, it is the mapping from $\mathbb{Z}^{2}$ to $T \cup\{E M P T Y\}$ where EMPTY is a special tile (null, null, null, null), indicating a tile is not present. For a configuration $C$, a tile $A=\left(\sigma_{n}, \sigma_{e}, \sigma_{s}, \sigma_{w}\right)$ is attachable at position $(i, j)$ iff $C(i, j)=$ EMPTY and $g\left(\sigma_{e}, w(C(i, j+1))\right)+g\left(\sigma_{n}, s(C(i+1, j))\right)+g\left(\sigma_{w}, e(C(i, j-1))\right)+$ $g\left(\sigma_{s}, n(C(i-1, j))\right) \geq \tau$, where indices $i$ and $j$ increase toward north and east directions, respectively.

Assembly takes place sequentially starting from a seed tile $s$ at a known position. One key aspect of this ATA model is that the glues are constant over time. For a given tile system, any assembly that can be obtained by starting from the seed and adding tiles one by one, is said to be produced. An assembly is called to be terminally 
produced if no further tiles can be added to it. The tile complexity of a shape $S$ is the size of the smallest tile set required to uniquely and terminally assemble $S$ under a given assembly model. One of the well-known results is that the tile complexity of self-assembly of a square of size $N \times N$ in ATA model is $\Theta\left(\frac{\log N}{\log \log N}\right)[3,32]$.

\subsection{Our Time-Dependent Glue (TDG) Model}

We propose a time-dependent glue model, which is built on the framework described above. In this model, the glue-strength between two tiles is dependent upon the time for which the two tiles have remained together.

Let $\tau$ be the temperature of the system. Tiles are defined as in the ATA model. However, in our model, glue strength function, $g$, is extended to contain a third argument that specifies the time for which the two sides of tiles are in contact. Formally speaking, $g$ is defined as $g: \Sigma \times \Sigma \times \mathbb{R} \rightarrow \mathbb{R}$.

In $g\left(\sigma, \sigma^{\prime}, t\right)$, the argument $t$ is the time for which two sides of the tiles with gluelabels $\sigma$ and $\sigma^{\prime}$ have been juxtaposed. For every pair $\left(\sigma, \sigma^{\prime}\right)$, the value $g\left(\sigma, \sigma^{\prime}, t\right)$ increases with $t$ up to a maximum limit and then takes a constant value determined by $\sigma$ and $\sigma^{\prime}$. We define the time when $g$ reaches this maximum as time for maximum strength and denote it as $\gamma: \Sigma \times \Sigma \rightarrow \mathbb{R}$. Note $g\left(\sigma, \sigma^{\prime}, t\right)=g\left(\sigma, \sigma^{\prime}, \gamma\left(\sigma, \sigma^{\prime}\right)\right)$ for $t \geq \gamma\left(\sigma, \sigma^{\prime}\right)$.

The minimum interaction time is a function $\mu: \Sigma \times \Sigma \rightarrow \mathbb{R}$. For every pair $\left(\sigma, \sigma^{\prime}\right)$, a function $\mu\left(\sigma, \sigma^{\prime}\right)$ is defined as the minimum time for which the two tiles with abutting glue symbols $\sigma$ and $\sigma^{\prime}$ stay together. If $g\left(\sigma, \sigma^{\prime}, \mu\left(\sigma, \sigma^{\prime}\right)\right) \geq \tau$, the two tiles will stay together; otherwise, they will separate if there is no other force holding them in their abutting positions. An example of glue-strength function is shown in Fig. 1. Intuitively speaking, $\mu$ serves as the minimum time required by the pads to decide whether they want to separate or remain joined. We further define $\mu(\sigma, n u l l)=0, \gamma(\sigma, n u l l)=0$, and $g(\sigma, n u l l, t)=0$.

Next, we give the justification and estimation of $\mu$ for a pair $\left(\sigma, \sigma^{\prime}\right)$ of glues. Let $g\left(\sigma, \sigma^{\prime}, t\right)$ be the glue-strength function. For more realistic estimation of $\mu$, consider

Fig. 1 Figure illustrates the concept of time-dependent glue strength, minimum interaction time, and time for maximum strength

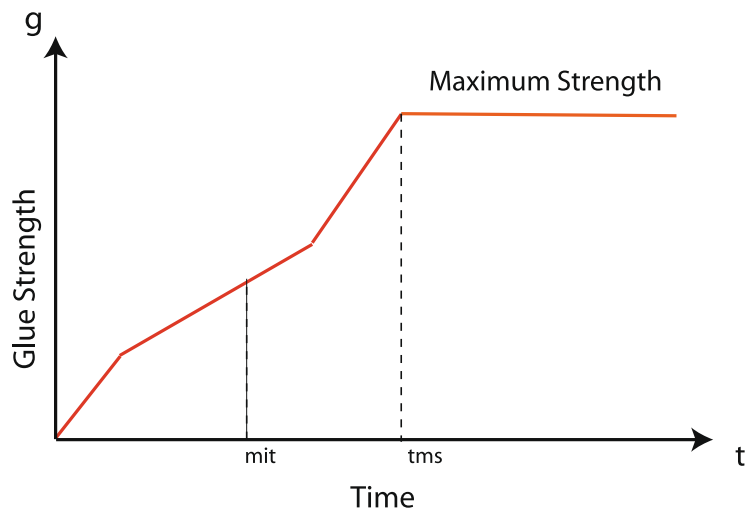


a physical system in which, in addition to association, dissociation reactions also occur. Let $p(b)$ be the probability of dissociation when the bond strength is $b$, where $p(b)$ can be determined using Winfree's kinetic model [40]. Assume that $f(t)$ be the probability that no dissociation takes place in the time interval $[0, t]$, and assume the time-interval $\delta t$ is so small that bond strength $g\left(\sigma, \sigma^{\prime}, t\right)$ does not change in the time-interval $t$ and $t+\delta t$. Then

$$
\begin{aligned}
f(t+\delta t) & =f(t) \cdot\left(1-p\left(g\left(\sigma, \sigma^{\prime}, t\right)\right)\right)^{\delta t}, \\
\frac{f(t+\delta t)}{f(t)} & =\left(1-p\left(g\left(\sigma, \sigma^{\prime}, t\right)\right)\right)^{\delta t}, \\
\frac{f(t+\delta t)}{f(t)} & =\exp \left(-\delta t \cdot p\left(g\left(\sigma, \sigma^{\prime}, t\right)\right)\right) .
\end{aligned}
$$

The probability that the dissociation takes place between time $t$ and $t+\delta t$ is given by $f(t) \cdot\left(1-\exp \left(-\delta t \cdot p\left(g\left(\sigma, \sigma^{\prime}, t\right)\right)\right)\right)$. Since $\mu$ is defined as the time for which two glues are expected to remain together once they come in contact, its expected value is

$$
E[\mu]=\lim _{\delta t \rightarrow 0} \sum_{t=0}^{\infty} t \cdot f(t) \cdot\left(1-\exp \left(-\delta t \cdot p\left(g\left(\sigma, \sigma^{\prime}, t\right)\right)\right)\right) .
$$

Hence, based on the knowledge of glue-strength function, it is possible to determine the expected minimum interaction time for a pair $\left(\sigma, \sigma^{\prime}\right)$. For simplicity, we will use the expected value of $\mu$ as the actual value of $\mu$ for a pair of glues $\left(\sigma, \sigma^{\prime}\right)$.

Next, we illustrate the time-dependent model with an example of the addition of a single tile to an aggregate. In a configuration $\mathrm{C}$, when a position $(i, j)$ becomes available for the addition of a tile $A$, it will stay at $(i, j)$ for a time interval $t_{0}$, where $t_{0}=\max \{\mu(e(A), w(C(i, j+1))), \mu(n(A), s(C(i+1, j))), \mu(w(A), e(C(i$, $j-1))), \mu(s(A), n(C(i-1, j)))\}$. Recall that our model requires that if two tiles ever come in contact, they will stay together till the minimum interaction time of the corresponding glues.

After this time interval $t_{0}$, if $g\left(e(A), w(C(i, j+1)), t_{0}\right)+g(n(A), s(C(i+$ $\left.1, j)), t_{0}\right)+g\left(w(A), e(C(i, j-1)), t_{0}\right)+g\left(s(A), n(C(i-1, j)), t_{0}\right)<\tau$, tile $A$ will detach; otherwise, $A$ will continue to stay at position $(i, j)$.

We describe in the next section a method to implement our model of timedependent glue strength with DNA tiles.

\section{Implementation of Time-Dependent Glue Model}

In this section, we propose an implementation of time-dependent glue model using DNA. Structurally, DNA is a long polymer of simple units called nucleotides, which are held together by a backbone made of sugars and phosphate groups. This backbone carries four types of bases (A, C, T, and G). These bases form complementary pairs ( $\mathrm{A}$ is complementary to $\mathrm{T}$ and $\mathrm{C}$ is complementary to $\mathrm{G}$ ) in a sense 


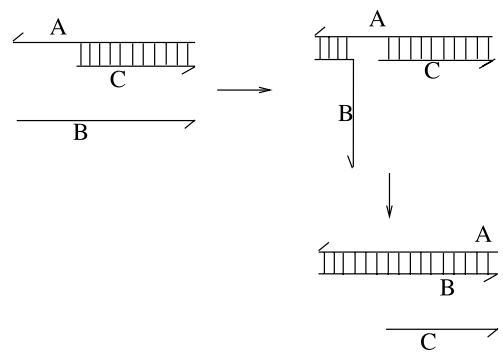

(a)

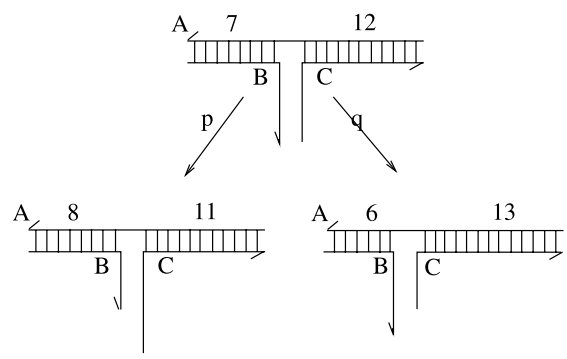

(b)

Fig. 2 (a) Illustrates the process of strand displacement. (b) Shows a single step of strand-displacement as single step of random walk. In (b), the numbers represent the number of DNA base pairs

that each base can form hydrogen bonds with the complementary base, also known as Watson-Crick base-pairing. The hydrogen bonding between complementary base pairs from two DNA strands results in their intertwining in the shape of a double helix, known as double stranded DNA (dsDNA). Individual separate strands are known as single stranded DNA (ssDNA). The direction of a DNA strand is defined in terms of its asymmetric ends, referred to as $5^{\prime}$ and $3^{\prime}$ ends. The $5^{\prime}$ end terminates at the phosphate group attached to the fifth carbon atom in the sugar ring, while the $3^{\prime}$ end terminates at hydroxyl group attached to the third carbon atom in the sugar-ring. In a double helix, direction of the nucleotides in one strand is opposite to their direction in the other strand.

The process of combining complementary, single stranded nucleic acids into a double stranded DNA molecule is called DNA hybridization. If the hydrogen bonds between the nucleotides in two hybridizing DNA strands build up sequentially, the total binding force between the two strands will increase with time up to the complete hybridization, which will provide a simple way of obtaining time-dependent glue strength between DNA tiles. However, even if we assume that the hybridization of two complementary DNA strands is instantaneous, we can design a multistep binding mechanism to implement the idea of time-dependent glue strength, which exploits the phenomenon of strand displacement.

Figure 2(a) illustrates the process of strand displacement in which strand $B$ displaces strand $C$ from strand $A$. Figure 2(b) illustrates one step during this process. At any time either the hybridization of $B$ with $A$ (and hence dehybridization of $C$ from $A$ ) or hybridization of $C$ with $A$ (and hence dehybridization of $B$ from $A$ ) can proceed with certain probability. Hence, we can model the strand displacement process as a random walk, with forward direction corresponding to hybridization between $B$ and $A$, and backward direction corresponding to hybridization between $C$ and $A$. A one-dimensional unbiased random walk is a process in which any step in forward or backward direction is taken with probability 0.5 independent of previous steps. The average straight-line distance between start and finish points of a one-dimensional random walk after $n$ steps is on the order of $\sqrt{n}$, and hence expected number of steps to cover a distance $n$ is $O\left(n^{2}\right)[15,17,28]$. In order to model 


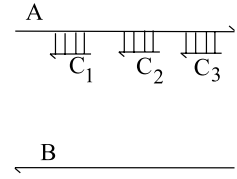

(a)

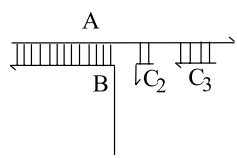

(e)

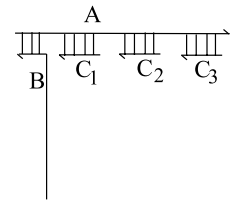

(b)

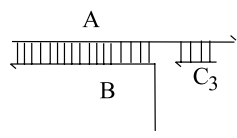

(f)

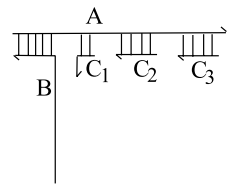

(c)

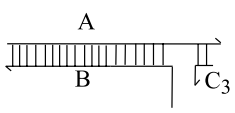

(g)

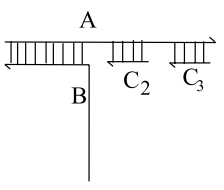

(d)

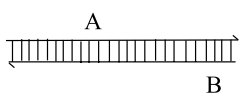

(h)

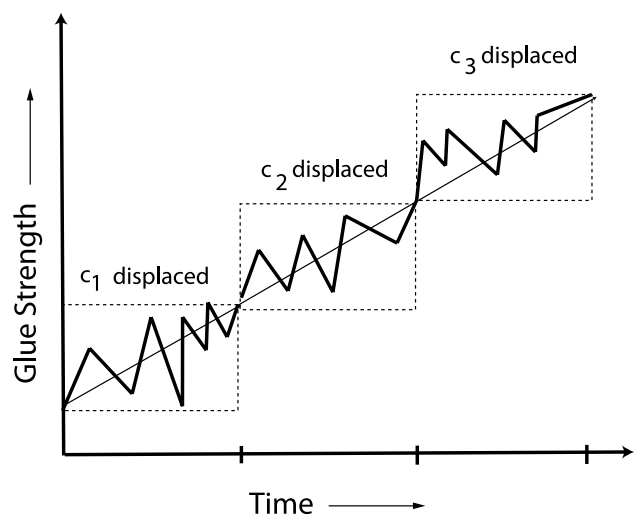

(i)

Fig. 3 (a) to (h) Illustrate a mechanism by which strand displacement reaction is used to implement time-dependent glue between two pads. They show step by step removal of $C_{i}$ 's by $B$ from $A$. In (i), an imaginary graph illustrates the variation of glue-strength between $A$ and $B$ w.r.t. time

the strand displacement, we can assume that the step length in this random walk is 1 base-pair long. Hence, if the length of $C$ is $n$ bases, the expected number of steps required for $B$ to replace $C$ is $O\left(n^{2}\right)$.

Next, we describe the design of the pads of DNA tiles with time-dependent glue using the above mechanism of strand displacement. To make the glue between pad $A$ and pad $B$ time-dependent, we need a construction similar to the one in Fig. 3(a). The strand representing pad $A$ has various smaller strands $\left(C_{i}\right.$ 's, called protector strands) hybridized to it as shown in Fig. 3(a). The strand $B$ will displace these protector strands $C_{i}$ sequentially.

Let the variable $\gamma$ here will be the time required for $B$ to displace all the $C_{i}$ 's. In the case when there are $k$ different small strands $C_{i}$ of length $n_{i}$ attached to $A, \gamma$ is $\sum_{i=1}^{k} n_{i}^{2}$.

Figure 3 gives the step by step illustration of the above process. The variation of glue strength between $A$ and $B$ is shown in Fig. 3(i). By controlling the length of 
various $C_{i}$ 's (i.e. $n_{1}, n_{2}, \ldots, n_{k}$ ), we can control the glue-strength function $g$ for a pair of tile-pads (or glues). Thus, we have shown a method to render the DNA tiles the characteristic of time-dependent glue strength.

An interesting property is that the individual strand displacement of $B$ against $C_{i}$ is modeled as an unbiased one dimensional random walk, but the complete process described above can be viewed as roughly monotonic. As shown in Fig. 3(i), the strength of the hybridization between strand $A$ and strand $B$ increases in a roughly monotonic fashion with the removal of every $C_{i}$. However during the individual competition between $B$ and $C_{i}$, the increase is not monotonic.

\section{Catalysis}

Catalysis is the phenomenon in which an external substance facilitates the reaction of other substances, without itself being used up in the process. A catalyst provides an alternative route of reaction where the activation energy is lower than the original chemical reaction and increase the reaction rate. Catalysts participate in reactions but are neither reactants nor products of the reaction they catalyze. The following question was posed by Adleman [2]: Can we model the process of catalysis in self-assembly of tiles? In this section, we present a model for catalysis in self-assembly of tiles using our time-dependent glue model. Now, consider a supertile $\mathcal{X}$ (composed of two attached tiles $C$ and $D$ ) and two single tiles $A$ and $B$ as shown in Fig. 4(a). We describe below how $\mathcal{X}$ can serve as a catalyst for the assembly of $A$ and $B$. Assume $t_{0}=\mu(e(A), w(B))$ such that $g\left(e(A), w(B), t_{0}\right)$ is less than the temperature $\tau$. Let $\mu(s(A), n(C))=\mu(s(B), n(D))=t_{1}>t_{0}$. Also, assume $g\left(s(A), n(C), t_{1}\right)+g\left(s(B), n(D), t_{1}\right)<\tau$ and $g\left(e(A), w(B), t_{1}\right) \geq \tau$.

The graph in Fig. 4(b) illustrates an example set of required conditions for the glue-strength functions in the system. $A \cdot B$ represents a tile $A$ bounded to a tile $B$.

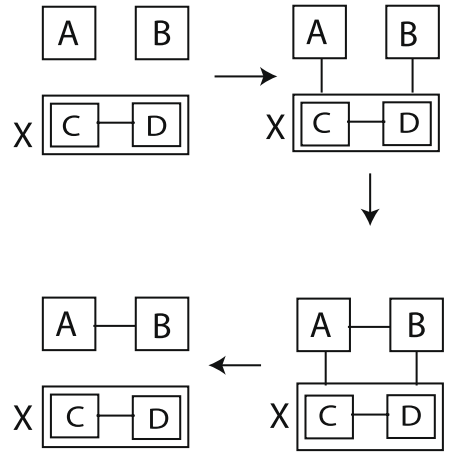

(a)

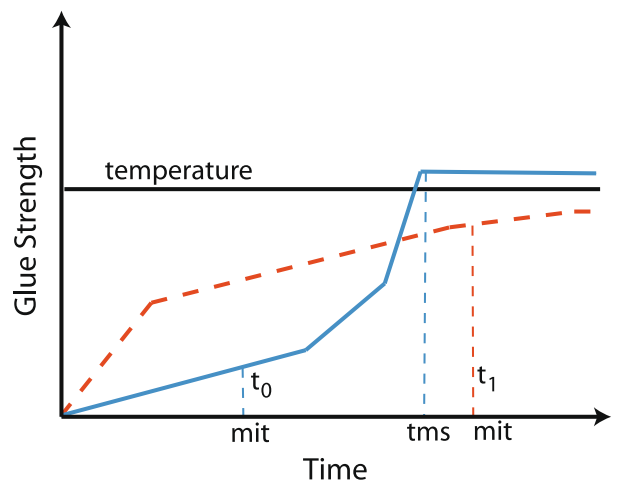

(b)

Fig. 4 (a) Shows catalyst $\mathcal{X}$ with the tiles $C$ and $D$ catalyzes the formation of $A \cdot B$. (b) Shows the conditions required for catalysis in terms of the glue-strength function. Solid line shows the plot of $g(e(A), w(B), t)$ and dashed line shows the plot of $g(s(A), n(C), t)+g(s(B), n(D), t)$ 
To show that $\mathcal{X}$ acts as a catalyst, we first show that without $\mathcal{X}$ stable $A \cdot B$ cannot form. Next, we show that $A \cdot B$ will form when $\mathcal{X}$ is present and $\mathcal{X}$ will be recovered unchanged after the formation of $A \cdot B$.

Without $\mathcal{X}$ in the system, $A$ and $B$ can only be held in neighboring positions for time $t_{0}=\mu(e(A), w(B))$, since $g\left(e(A), w(B), t_{0}\right)<\tau$. Hence, at $t_{0}, A$ and $B$ will fall apart.

However, in the presence of $\mathcal{X}$, the situation changes. Supertile $\mathcal{X}$ has two neighboring tiles $C$ and $D$. Tiles $A$ and $B$ attach themselves to $C$ and $D$ as shown in Fig. 4(a). Since we let $\mu(s(A), n(C))=\mu(s(B), n(D))=t_{1}>t_{0}$, tiles $A$ and $B$ are held in the same position for time $t_{1}$. By our construction, as shown in Fig. 4(b), the following two events will occur at time $t_{1}$ :

- At $t_{1}$, the glue strength between $A$ and $B$ is $g\left(e(A), w(B), t_{1}\right) \geq \tau$, and hence $A$ and $B$ will be glued together. That is, in the presence of $\mathcal{X}, A$ and $B$ remain together for a longer time, producing stably glued $A \cdot B$.

- At $t_{1}$, the total glue strength between $A \cdot B$ and $\mathcal{X}$ is $g\left(s(A), n(C), t_{1}\right)+$ $g\left(s(B), n(D), t_{1}\right)<\tau$, and the glued $A \cdot B$ will fall off $\mathcal{X} . \mathcal{X}$ is recovered unchanged from the reaction and the catalysis is complete. Now, $\mathcal{X}$ is ready to catalyze other copies of $A$ and $B$.

Note that if only $A$ (resp. $B$ ) comes in to attach with $C$ (resp. $D$ ), it will fall off at the end of time $\mu(s(A), n(C))$ (resp. $\mu(s(B), n(D))$ ). If assembled $A \cdot B$ comes in, it will also fall off, at time $t_{1}$. These two reactions are futile reactions, and do not block the desired catalysis reaction. However, as the concentration of $A \cdot B$ increases and the concentration of unattached $A$ and $B$ decreases, the catalysis efficiency of $\mathcal{X}$ will decrease due to the increased probability of the occurrence of futile reaction between $A \cdot B$ and $C \cdot D$.

\section{Self-replication}

Self-replication process is one of the fundamental process of nature, in which a system creates copies of itself. For example, DNA is self-replicated during cell division and is transmitted to offspring during reproduction. A material device that can self-replicate is ambition of many engineering disciplines. The biggest incentive is to achieve a low manufacturing cost because self-replication avoids the costs of labor, capital, and distribution in conventional manufactured goods. In an evolving field like nanotechnology, manufacturing costs of molecular machines can become extremely large in the absence of self-replication. We discuss below an approach to model the process of self-replication in DNA tiles assembly using our time-dependent glue model.

Our approach is built on the above described process of catalysis: a product $A \cdot B$ catalyzes the formation of $C \cdot D$, which in turn catalyzes the formation of $A \cdot B$, and hence an exponential growth of self-replicated $A \cdot B$ and $C \cdot D$ takes place. 
More precisely, let $t_{0}<t_{1}$, and consider tiles $A, B, C$, and $D$, such that

$$
\begin{array}{r}
\mu(e(A), w(B))=\mu(e(C), w(D))=t_{0}, \\
\mu(s(A), n(C))=\mu(s(B), n(D))=t_{1}, \\
g\left(e(A), w(B), t_{0}\right)=g\left(e(C), w(D), t_{0}\right)<\tau, \\
g\left(e(A), w(B), t_{1}\right)=g\left(e(C), w(D), t_{1}\right)>\tau, \\
g\left(s(A), n(C), t_{1}\right)+g\left(s(B), n(D), t_{1}\right)<\tau .
\end{array}
$$

A system containing these four types of tiles has two states.

State 1. If there is no template $A \cdot B$ or $C \cdot D$ in the system, no assembled supertile exists since no two tiles can be held together long enough to form strong enough glue between them such that they become stably glued. Since $\mu(e(A), w(B))=$ $\mu(e(C), w(D))=t_{0}$ and $g\left(e(A), w(B), t_{0}\right)=g\left(e(C), w(D), t_{0}\right)<\tau$, neither stable $A \cdot B$ nor stable $C \cdot D$ can form. Similarly, $\mu(s(A), n(C))=\mu(s(B), n(D))=t_{1}$, $g\left(s(A), n(C), t_{1}\right)<\tau$, and $g\left(s(B), n(D), t_{1}\right)<\tau$ implies that neither stable $A \cdot C$ nor stable $B \cdot D$ can form.

State 2. In contrast, if there is an initial copy of stable $A \cdot B$ in the system, selfreplication occurs as follows. $A \cdot B$ serves as catalyst for the formation of $C \cdot D$, and $C \cdot D$ and $A \cdot B$ separate from each other at the end of the catalysis period, as described in Sect. 4; in turn, $C \cdot D$ serves as catalyst for the formation of $A \cdot B$. Thus, we have a classical self-replication system: one makes a copy of itself via its complement. The number of the initial template $(A \cdot B)$ and its complement $(C \cdot D)$ grows exponentially in such a system as long as there are sufficient numbers of free $A, B, C$, and $D$ tiles to be made into pairs.

Hence, if the system is in state 1 , it needs a triggering activity (formation of a stable $A \cdot B$ or $C \cdot D$ ) to go into state 2 . Once the system is in state 2 , it starts

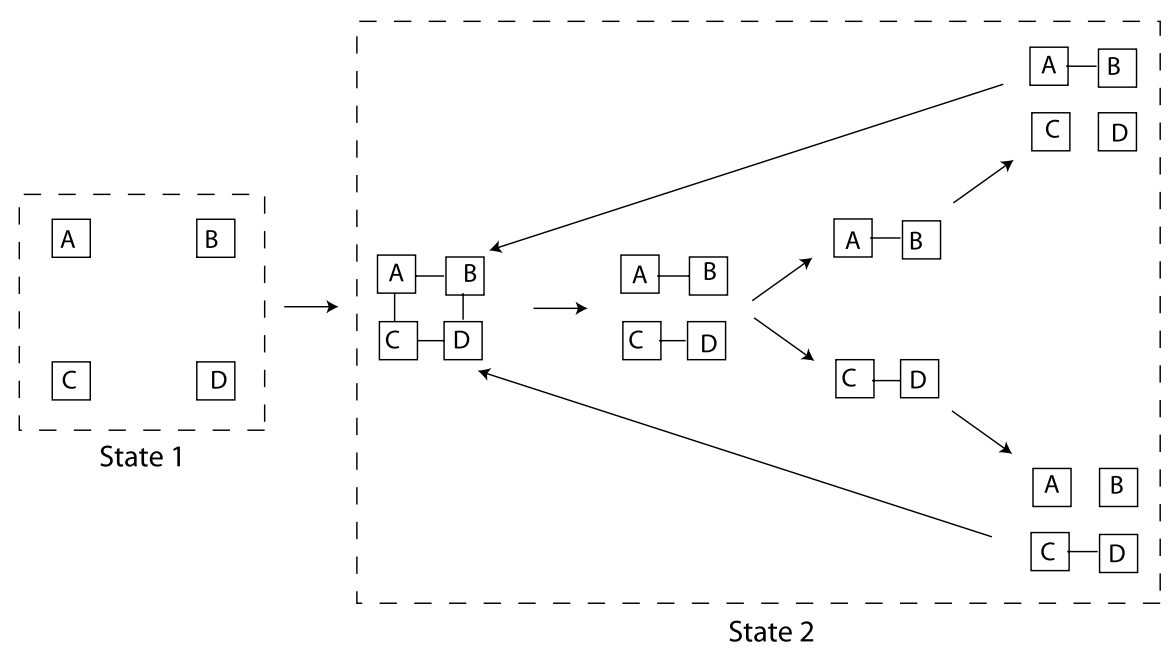

Fig. 5 A schematic of self-replication 
the self-replication process. Figure 5 illustrates the process of self-replication in the assembly of tiles.

If the system is in state 1 , then the triggering activity (formation of a stable $A \cdot B$ or $C \cdot D$ ) can take place only if $A, B, C, D$ coposition themselves so that the east side of $A$ faces the west side of $B$ and the south side of $A$ faces the north side of $C$, and at the same time the south side of $B$ faces the north side of $D$. In such a situation, $A$ and $C$ will remain abutted until time $t_{1}, B$ and $D$ will remain abutted until time $t_{1}$, and $A$ and $B$ (and $C$ and $D$ ) might also remain together for time $t_{1}$, producing stable $A \cdot B$ and stable $C \cdot D$. And this will bring the system to state 2 . Such copositioning of 4 tiles is a very low probability event. However, among other conditions, appropriate copositioning of unstable $A \cdot B$ and unstable $C \cdot D$, or unstable $A \cdot C$ and unstable $B \cdot D$ can also perturb a system in state 1 and triggers tremendous changes by bringing the system to state 2 where self-replication occurs.

\section{Tile Complexity Results}

\subsection{Tile Complexity Results for Thin Rectangles}

In the ATA model, the tile complexity of assembling an $N \times N$ square is $\Theta\left(\frac{\log N}{\log \log N}\right)$ $[3,32]$. It is also known that the upper bound on the tile complexity of assembling a $k \times N$ rectangle in the ATA model is $O\left(k+N^{1 / k}\right)$ and that the lower bound on tile complexity of assembling a $k \times N$ rectangle is $\Omega\left(\frac{N^{1 / k}}{k}\right)$ [6]. For small values of $k$, this lower-bound is asymptotically larger than $O\left(\frac{\log N}{\log \log N}\right)$. Here, we claim that in our model, as in the multitemperature model defined in [6], a $k \times N$ rectangle can be self-assembled using $O\left(\frac{\log N}{\log \log N}\right)$ types of tiles, even for small values of $k$. The proof technique follows the same spirit as in [6].

Theorem 1 In the time-dependent glue model, the tile complexity of self-assembling a $k \times N$ rectangle for an arbitrary integer $k \geq 2$ is $O\left(\frac{\log N}{\log \log N}\right)$.

Proof The tile complexity of self-assembling a $k \times N$ rectangle is $O\left(N^{\frac{1}{k}}+k\right)$ for the ATA model [6]. In the time-dependent glue model, we can use the similar idea as in [6] to reduce the tile complexity of assembling thin rectangles. For given $k$ and $N$, build a $j \times N$ rectangle with $j>k$ such that the glues among the first $k$ rows become strong after their $\mu$ (minimum interaction time), while the glues among the last $j-k$ rows do not become as strong. First, $k$ rows are called stable rows, and last $j-k$ rows are called volatile rows. As such, these $j-k$ volatile rows, will disassemble from the assembly after certain time leaving the target $k \times N$ rectangle consisting of only the stable rows.

The tile set required to accomplish this construction is shown in Fig. 6, which is similar to the one used in [6]. For more detailed illustration of this tile set, refer to [6]. First, a $j$-digit $m$-base counter is assembled as follows. Starting from the west 
Hairpin Tiles,

Return probes and Probes

Normal Tiles

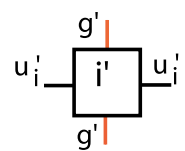

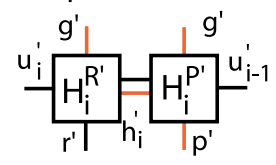

$u_{0}^{S_{j-1}^{\prime}}$

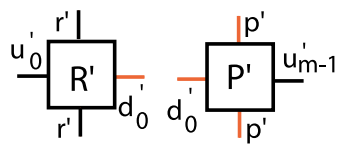
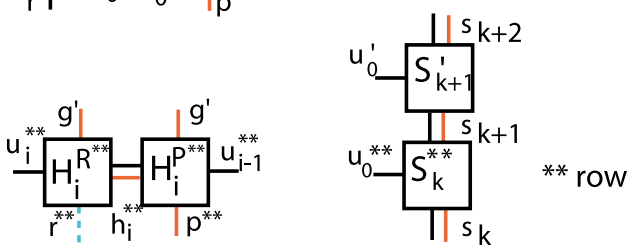

$u_{g^{* * *}}^{u_{i^{* *}}^{g^{\prime}}} u_{i}^{* *}$
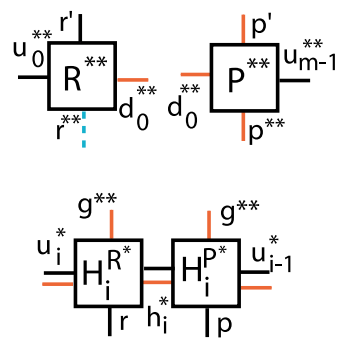

$u_{i}^{u_{i}^{*}} \underbrace{i^{* *}} u_{i}^{*}$
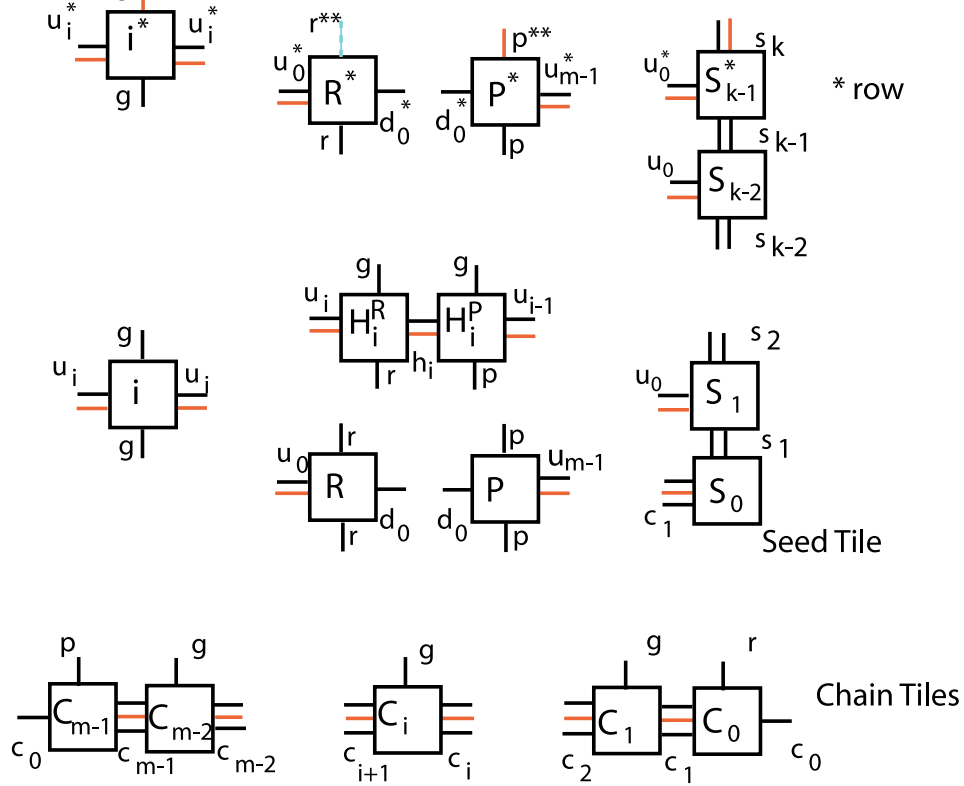

Fig. 6 Tile set to construct a $k \times N$ rectangle using only $O\left(N^{1 / j}+j\right)$ tiles. The glue-strength functions of red, blue, and black glues are defined in the proof 
edge of the seed tile, a chain of length $m$ is formed in the first row using $m$ chain tiles. At the same time, tiles in the seed column also start assembling. It should be noted that first $k$ tiles in the seed column have sufficient glue strength and they are stable. Now starting from their west edges, the 0 normal tiles start filling the $m-1$ columns in the upper rows. Then the hairpin tiles $H_{1}^{P}$ and $H_{1}^{R}$ assemble in the second row, which causes the assembly of further $m$ chain tiles in the first row, and the assembly of 1 normal tiles in the second row (and 0 normal tiles in the upper rows) in the next section of $m$ columns. Generally speaking, whenever a $C_{m-1}$ chain tile is assembled in the first row, probe tiles in the upper rows are assembled until reaching a row that does not contain an $m-1$ normal tile. In such a row, the appropriate hairpin tiles are assembled and this further propagates the assembly of return probe tiles downward until the first row is reached, where a $C_{0}$ chain tile gets assembled. This again starts an assembly of a chain of length $m$. The whole process is repeated until a $j \times m^{j}$ rectangle is assembled.

Next, we describe our modifications which are required for the $j-k$ upper volatile rows to get disassembled after the complete assembly of the $j \times m^{j}$ rectangle. First of all, we need to have a special $(k+1)$-th row $(* *$ row), which will assemble to the north of the $k$ th row ( $*$ row), as shown in Fig. 6.

The operating temperature $\tau=2$. Assume that for all glue-types, $\mu=t_{0}$ and $\gamma=t_{1}$. There are three kinds of glues shown in Fig. 6: black, red, and blue. Assume that the glue-strength function for a single black glue is $g_{\text {black }}(t)$, a single red glue is $g_{\text {red }}(t)$, and a single blue glue is $g_{\text {blue }}(t)$. They are defined as

$$
\begin{gathered}
g_{\text {black }}(t)= \begin{cases}\frac{4 t}{5 t_{0}}, & t<t_{0}, \\
\frac{4}{5}+\frac{t-t_{0}}{5\left(t_{1}-t_{0}\right)}, & t_{0} \leq t<t_{1}, \\
1, & t \geq t_{1},\end{cases} \\
g_{\text {red }}(t)= \begin{cases}\frac{2 t}{5 t_{0}}, & t<t_{0}, \\
\frac{2}{5}+\frac{t-t_{0}}{10\left(t_{1}-t_{0}\right)} & t_{0} \leq t<t_{1}, \\
\frac{1}{2} & t \geq t_{1},\end{cases} \\
g_{\text {blue }}(t)=\left\{\begin{array}{lll}
\frac{2 t}{5 t_{0}}, & t<t_{0}, \\
\frac{2}{5}, & t \geq t_{0} .
\end{array}\right.
\end{gathered}
$$

Multiple glues shown on the same side of a tile in Fig. 6 are additive. For example, the glue-strength between $C_{i}$ and $C_{i+1}(0 \leq i \leq m-2)$ is $2 g_{\text {black }}(t)+g_{\text {red }}(t)$.

This system will start assembling like a base $N^{1 / j}$ counter of $j$ digits, as briefed above and detailed in [3, 6]. It will first construct a rectangle of size $j \times N$ using $N^{1 / j}+j$ type of tiles. Once the rectangle is complete, the tile on the north-west corner will start the required disassembly of the upper $(j-k)$ volatile rows, which results in the formation of a $k \times N$ rectangle. We call these two phases Assembly phase and Disassembly phase, respectively, and describe them below.

Assembly Phase. In the assembly phase, we aim at constructing a $j \times N$ rectangle. In the time dependent model, the assembly proceeds as in the ATA model until 
the assembly of $P^{*}$ tile in the $k$ th row (the distinguished $*$ row). At this point, an $H^{R^{* *}}$ tile is required to get assembled. However, when the $H^{R^{* *}}$ tile is assembled in the $(k+1)$-th row, the total support on $H^{R^{* *}}$ from its east neighbor is only $\frac{4}{5}+\frac{2}{5}<2$ at the end of $\mu$. Thus, $H^{R^{* *}}$ must obtain additional support; otherwise, it will get disassembled, blocking the desired assembly process. The additional support comes both from its south neighbor and its west neighbor. (1) On the south front, tile $R^{*}$ can arrive and be incorporated in the $k$ th row (the distinguished $*$ row) of the assembly. It holds $H^{R^{* *}}$ for another time interval of $\mu$ and provides a support of $\frac{2}{5}$. Further note that during this second interval, an $R$ tile can be assembled in the $(k-1)$-th row, and the $R^{*}$ tile in the $k$ th row will then have support 2 at $\mu$, and hence stay attached. In addition, tile $R$ has support 2 at $\mu$, so it will also stay attached. Regarding $H^{R^{* *}}$, the end result is that it receives an additional stable support $\frac{2}{5}$ from its south neighbor. However, the maximum support from both the south and the east is at most $1+\frac{1}{2}+\frac{2}{5}$, which is still less than $\tau=2$. Fortunately, additional rescue comes from the west. (2) On the west front, an $i^{* *}$ tile can get attached to $H^{R^{* *}}$, and stabilize it by raising its total support above 2 . However, this support is insufficient, in the sense that $i^{* *}$ itself needs additional support from its own west and south neighbors to stay attached. If this support cannot come in time, that is, before $\mu, i^{* *}$ will get disassembled, in turn causing the disassembly of $H^{R^{* *}}$. The key observation here is that this assembly/disassembly is a reversible dynamic process: the disassembly may stop and start going backward (i.e., assembling again) at any point. Thus, in a dynamic, reversible fashion, the target structure of the assembly phase, namely the $j \times N$ rectangle, can be eventually constructed.

The above added complication is due to the fact that we require the $H^{R^{* *}}$ tiles in the $(k+1)$-th row to get a total support of $<2$ from the south and the east. This is crucial because during the subsequent disassembly phase (as we describe next) the desired disassembly can only carry through if the total support of each volatile tile from the south and the east is $<2$.

Disassembly Phase. In the disassembly phase, we will remove the $j-k$ volatile rows, and reach the final target structure, a $k \times N$ rectangle. Once the $j \times N$ rectangle is complete, the tile $T$ at the north-west corner ( $P^{\prime}$ tile in the $j$ th row) initiates the disassembly. When the $\mu$ of the glue-pairs between tile $T$ and its neighbors is over, tile $T$ will get detached because the total glue strength that it has accumulated is $\frac{4}{5}+\frac{2}{5}<\tau=2$. Note that unlike the above case for $H^{R^{* *}}$, no additional support can come from the west for tile $T$ since $T$ is the west-most tiles. As such, $T$ is doomed to get disassembled. With $T$ gone, $T$ 's east neighbor will get removed next, since it now has a total glue strength $\leq 1+\frac{1}{2}<\tau$. Similarly, all the tiles in this row will get removed one by one, followed by the removal of the tiles in the next row (south row). Such disassembly of the tiles continues until we are left with the target rectangle of size $k \times N$, whose constituent tiles, at this stage, all have a total glue strength no less than $\tau=2$, and hence stay stably attached.

Note that, similar as in the assembly phase, the volatile tiles that just got removed might come back. But again, ultimately they will have to all fall off (after the $\mu$ ), and produce the desired $k \times N$ rectangle. 
Concluding the Proof. We can construct a $k \times N$ rectangle using $O\left(N^{1 / j}+j\right)$ type of tiles (where $j>k$ ). As in [6], it can be reduced to $O\left(\frac{\log N}{\log \log N}\right)$ by choosing $j=\frac{\log N}{\log \log N-\log \log \log N}$.

\subsection{Further Tiling Assemblies for Interesting Shapes}

Thin rectangles can serve as building blocks for the construction of many other interesting shapes. One example is a square of size $N \times N$ with a large square hole of size $k \times k$ (for $k \sim N$ ). Under the ATA model, the lower bound can be shown to be $\Omega\left(\frac{(k)^{\frac{2}{N-k}}}{N-k}\right)$ by a lower bound argument similar to the one in [6]. Note that as $N-k$ decreases, i.e., the square hole in the square increases, the lower bound increases. In the case when $N-k$ is smaller than $\frac{\log N}{\log \log N-\log \log \log N}$, the lower bound is more than $\frac{\log N}{\log \log N}$. In the case when $N-k$ is a small constant, the complexity is almost $N^{c}$, where $c$ is some constant $<1$. However, in the time-dependent model, the tile complexity of this shape can be reduced to $O\left(\frac{\log k}{\log \log k}\right)$ even for small values of $N-k$, using our thin rectangle construction.

The basic idea is quite simple. We sequentially grow four different thin rectangles in four different directions: one rectangle northward, one westward, one southward, and one eastward. The dimensions of each of these rectangles is $\left(\frac{N-k-2}{2}\right) \times$ $(k+2)$. They will make up the major part of the square's sides as shown in Fig. 7(a). The required tile set consists of four different groups of tile sets: each one growing a $\left(\frac{N-k-2}{2}\right) \times(k+2)$ rectangle in one direction. Each of these rectangles can be constructed by $O\left(\frac{\log k}{\log \log k}\right)$ types of tiles as discussed in the proof of Theorem 1 . We

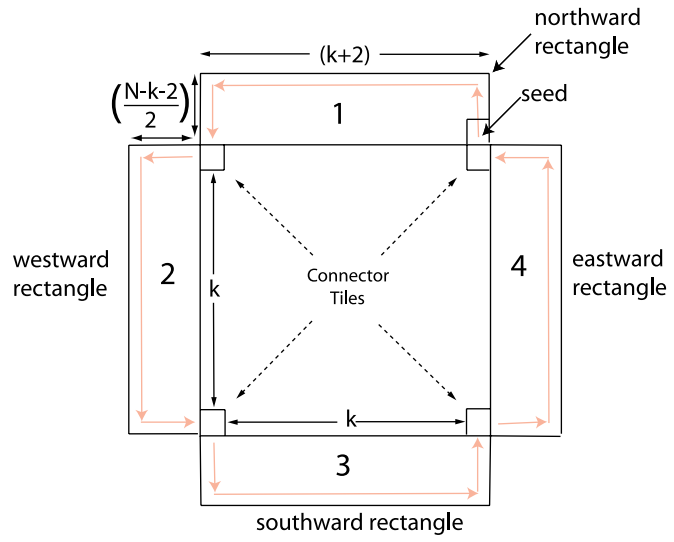

(a)

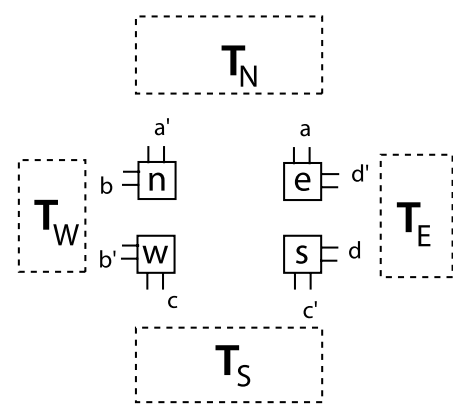

(b)

Fig. 7 (a) Direction of the red arrow shows the direction of construction of a square with a hole, starting from the indicated seed. (b) A complete tile set for the square with hole. Sets $T_{N}, T_{S}, T_{W}$, $T_{E}$ are shown in Figs. 8, 9, and 10 


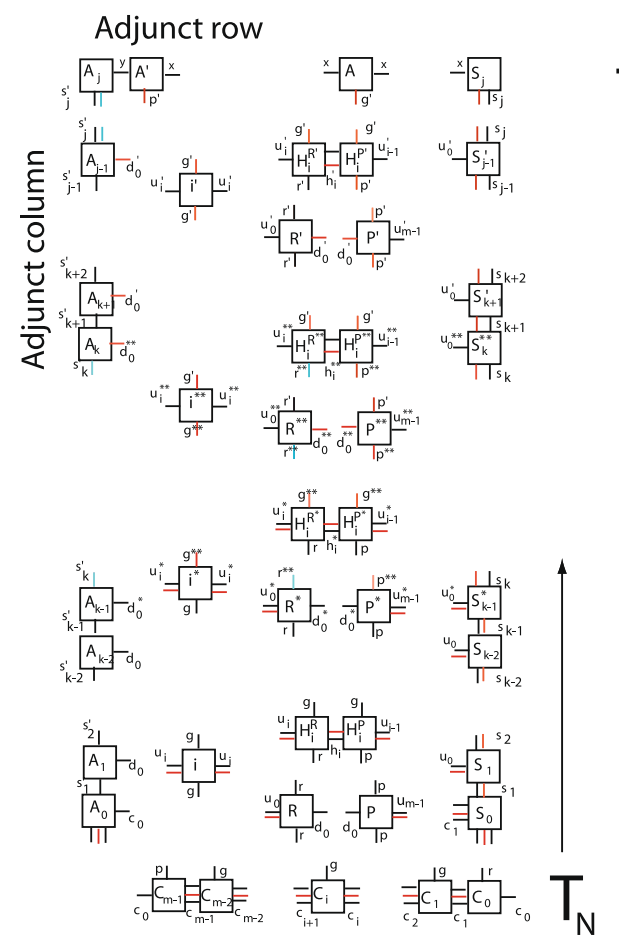

(a)

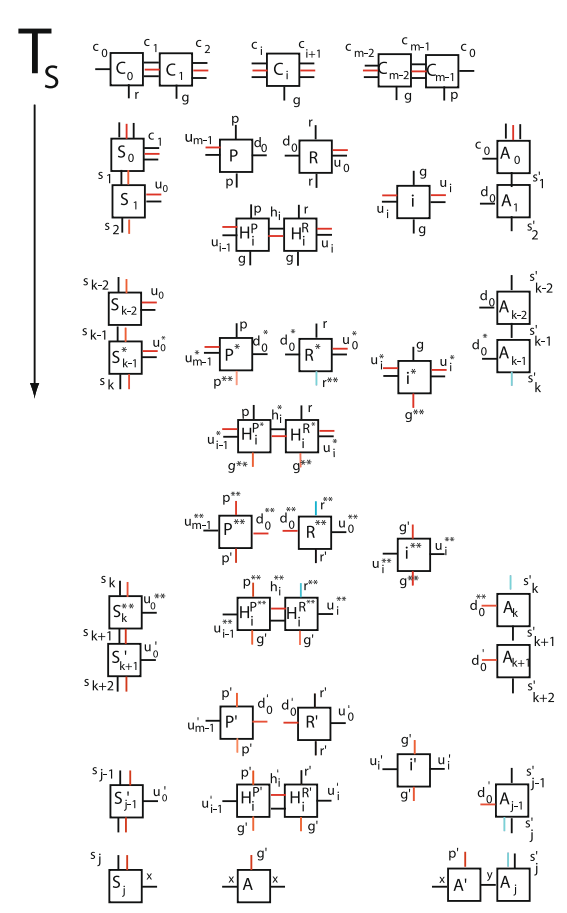

(b)

Fig. 8 (a) Displays the tiles from the sets $T_{N}$ required for the construction of $N \times N$ square with a hole of size $k \times k$ in the center. (b) Displays the tiles from the sets $T_{S}$ required for the construction of $N \times N$ square with a hole of size $k \times k$ in the center. It should be noted that symbols in (a), (b), Figs. 9, and 10 are from different namespaces. It means that a glue-symbol $x$ in $T_{N}$ is different from a glue-symbol $x$ in $T_{S}, T_{W}$, or $T_{E}$, and they cannot interact

refer to these groups of tile sets as $T_{N}, T_{W}, T_{S}$, and $T_{E}$ (Fig. 7 (b)). The complete details of tile sets $T_{N}, T_{W}, T_{S}$, and $T_{E}$ are shown in Figs. 8, 9, and 10.

As shown in the center in Fig. 7(b), we need some additional tiles (tiles $n, e, s$, and $w$ ) to connect these four different rectangles with each other in order to complete the desired square with a hole. We call them connector tiles. Note that the glues on the sides of connector tiles match with the glues of seed tiles of the corresponding rectangles. After the completion of one rectangle, the corresponding connector tile should assemble and provide a path for the assembly of another rectangle. For example, the assembly of the connector tile $n$ takes place after the assembly of the west-most column of the northward rectangle from the tile set $T_{N}$, and triggers the assembly of the westward rectangle.

In each of the thin rectangles, a special row and a special column is needed that can assist the assembly of the corresponding connector tile. We call these special rows and columns as adjunct row and adjunct column. The tiles required for the assembly of the adjunct row and column in the northward rectangle are shown in 


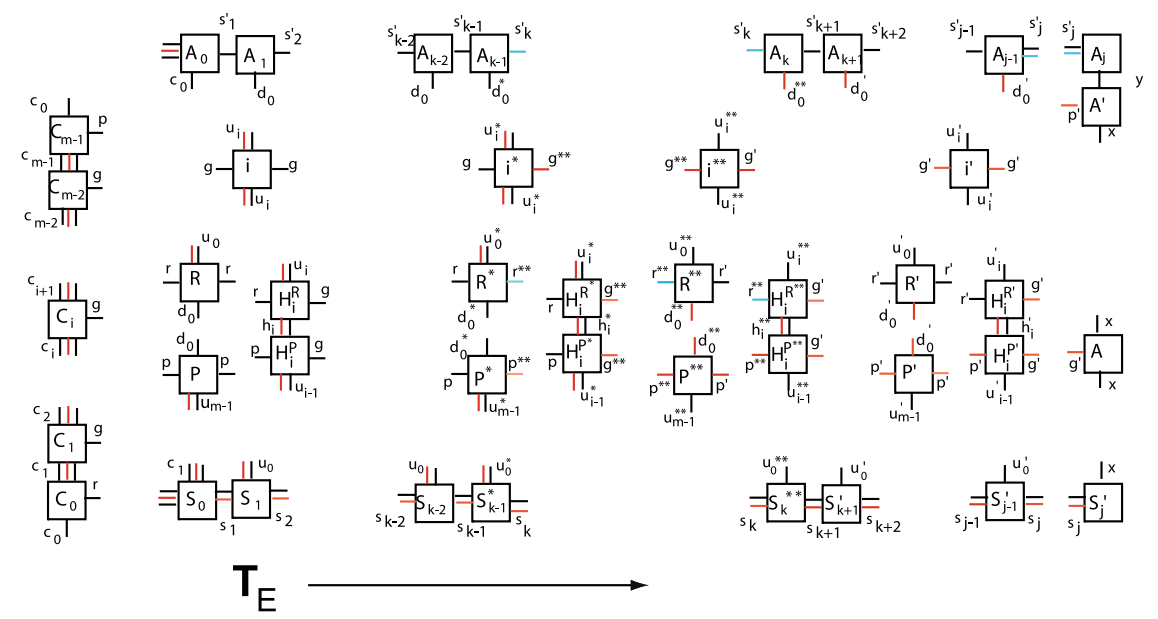

Fig. 9 Figure displays the tiles from the sets $T_{N}$ and $T_{S}$ required for the construction of $N \times N$ square with a hole of size $k \times k$ in the center

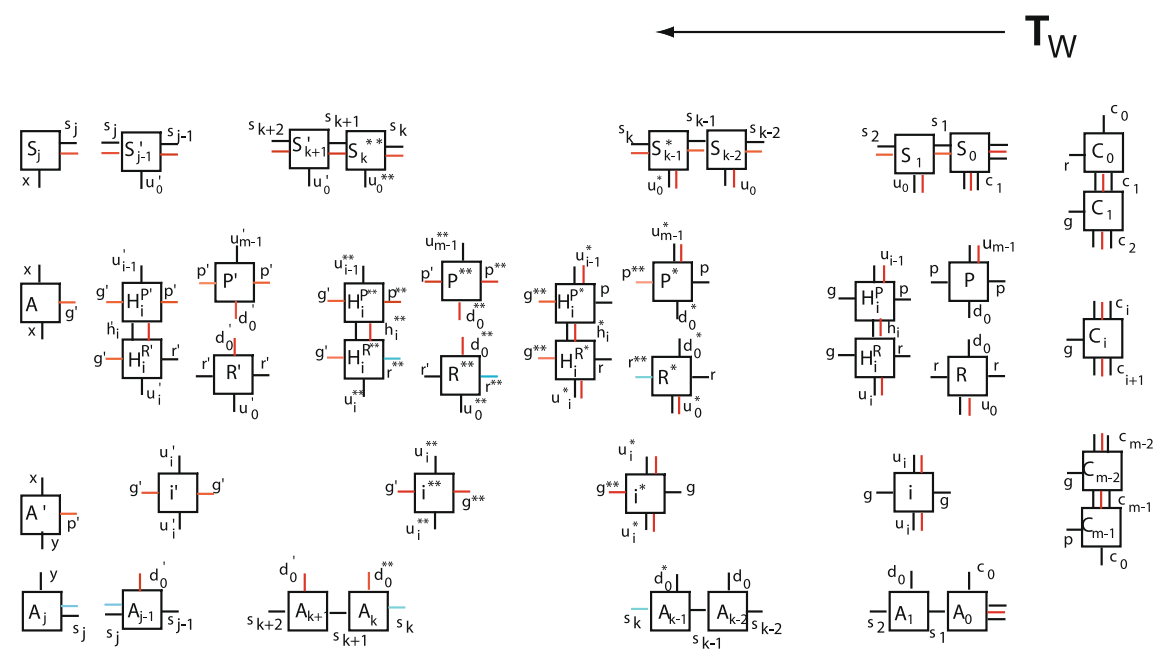

Fig. 10 Figure displays the tiles from the set $T_{W}$ required for the construction of $N \times N$ square with a hole of size $k \times k$ in the center

Fig. 8. Note that the glues on the sides of these tiles are designed in such a way that they do not inhibit the disassembly phase in the construction of the corresponding thin rectangle.

Finally, we have gaps at the four corners this $N \times N$ square, and a $(k+2) \times$ $(k+2)$ square hole in the center with exactly one tile present at each corner of the hole (Fig. 7). A constant number of type of tiles, referred to as filler tiles, will be 
needed to fill in these gaps, and obtain an $N \times N$ square with a $k \times k$ hole at its center.

The complete tile set $T_{N}$ is the tile set described in the proof of Theorem 1 along with the tiles for adjunct row and column. The boundary tiles in $T_{N}$ are modified slightly so that they can assist the assembly of appropriate filler tiles when required. Tile set $T_{W}$ is formed by rotating every tile of $T_{N}$ anticlockwise by $90^{\circ}$. It should be noted that a totally disjoint set of symbols for the glues should be used in $T_{W}$ to avoid any interaction with the tiles in $T_{N}$. Similarly, the tile sets $T_{S}$ and $T_{E}$ can be obtained by further anticlockwise rotation of $T_{W}$ by $90^{\circ}$ and $180^{\circ}$, respectively.

Thus, the total number of tiles required is the sum of tiles required for each of the four thin rectangles, four connector tiles, constant number of filler tiles, and the tiles for adjunct row and column in each of the four rectangles. This is upper bounded by $O\left(\frac{\log k}{\log \log k}\right)$. The assembly will grow in the manner shown in Fig. 7(a). Assuming without loss of generality that the seed is the seed tile of rectangle 1 . Then first rectangle 1 will be constructed; then the connector to rectangle 1 and 2 will assemble; then rectangle 2 will assemble; then connector to rectangle 2 and 3; then rectangle 3; then connector to 3 and 4; finally rectangle 4 will get assembled. It should be noted that the filler tiles can assemble anytime during the assembly, whenever they get enough support to hold them.

\section{Discussion and Future Work}

In this paper, we defined a model in which the glue strength between tiles depends upon the time they have been abutting each other. Under this model, we demonstrate and analyze catalysis and self-replication, and show how to construct a thin $k \times N$ rectangle using $O\left(\frac{\log N}{\log \log N}\right)$ tiles for constant $k>0$. The upper bound on assembling a thin rectangle is obtained by applying similar assembly strategy as in the multitemperature model [6]. Thus, an interesting question is whether the multitemperature model can be simulated using our time-dependent model. It is also an open problem if under our model the lower bound of $\Omega\left(\frac{\log N}{\log \log N}\right)$ for the tile complexity of an $N \times N$ square can be further improved.

Another interesting direction is to study the kinetics of the catalysis and selfreplication analytically. Winfree's kinetic model [40] can be used to study them, but the challenge here is that the rate constant for the dissociation for a particular species varies with time because of changing glue strengths of its bonds. This makes the analytical study hard. However, these catalytic and self-replicating systems can be modeled as a continuous time Markov chain, and studied using computer simulation to obtain empirical results.

Acknowledgements The work is supported by NSF Grants CCF-0523555 and CCF-0432038.

\section{References}

1. http://mrsec.wisc.edu/edetc/selfassembly/ 
2. Adleman L (2000) Towards a mathematical theory of self-assembly. Tech Rep 00-722, University of Southern California

3. Adleman L, Cheng Q, Goel A, Huang M (2001) Running time and program size for selfassembled squares. In: Proceedings of the thirty-third annual ACM symposium on theory of computing. ACM, New York, pp 740-748

4. Adleman L, Cheng Q, Goel A, Huang M, Kempe D, de Espans P, Rothemund P (2002) Combinatorial optimization problems in self-assembly. In: Proceedings of the thirty-fourth annual ACM symposium on theory of computing. ACM, New York, pp 23-32

5. Adleman L, Kari J, Kari L, Reishus D (2002) On the decidability of self-assembly of infinite ribbons. In: Proceedings of the 43rd symposium on foundations of computer science, pp 530 537

6. Aggarwal G, Cheng Q, Goldwasser MH, Kao M, de Espanes PM, Schweller RT (2005) Complexities for generalized models of self-assembly. SIAM J Comput 24:1493-1515

7. Angelov S, Khanna S, Visontai M (2008) On the complexity of graph self-assembly in accretive systems. Nat Comput Int J Arch 7:183-201

8. Bowden N, Terfort A, Carbeck J, Whitesides G (1997) Self-assembly of mesoscale objects into ordered two-dimensional arrays. Science 276(11):233-235

9. Bruinsma R, Gelbart W, Reguera D, Rudnick J, Zandi R (2003) Viral self-assembly as a thermodynamic process. Phys Rev Lett 90(24):248101

10. Chelyapov N, Brun Y, Gopalkrishnan M, Reishus D, Shaw B, Adleman L (2004) DNA triangles and self-assembled hexagonal tilings. J Am Chem Soc 126:924-925

11. Chen H, Cheng Q, Goel A, Huang M, de Espanes P (2004) Invadable self-assembly: combining robustness with efficiency. In: Proceedings of the 15th annual ACM-SIAM symposium on discrete algorithms (SODA), pp 890-899

12. Cheng Q, de Espanes P (2003) Resolving two open problems in the self-assembly of squares. Tech Rep 03-793, University of Southern California

13. Cheng Q, Goel A, Moisset P (2004) Optimal self-assembly of counters at temperature two. In: Proceedings of the first conference on foundations of nanoscience: self-assembled architectures and devices

14. Cook M, Rothemund PWK, Winfree E (2004) Self-assembled circuit patterns. In: DNA based computers 9. Lecture notes in computer science, vol 2943. Springer, Berlin, pp 91-107

15. Feller W (1968) An introduction to probability theory and its applications, vol 1

16. Fujibayashi K, Murata S (2005) A method for error suppression for self-assembling DNA tiles. In: Lecture notes in computer science, vol 3384. Springer, Berlin, pp 113-127

17. Hughes BD (1995) Random walks and random environments, vol 1: random walks. Oxford University Press, New York

18. Kao M, Schweller R (2006) Reduce complexity for tile self-assembly through temperature programming. In: Proceedings of 17th annual ACM-SIAM symposium on discrete algorithms (SODA). ACM, New York, pp 571-580

19. Klavins E (2004) Directed self-assembly using graph grammars. In: Foundations of nanoscience: self assembled architectures and devices, Snowbird, UT

20. Klavins E, Ghrist R, Lipsky D (2004) Graph grammars for self-assembling robotic systems. In: Proceedings of the international conference on robotics and automation

21. LaBean TH, Yan H, Kopatsch J, Liu F, Winfree E, Reif JH, Seeman NC (2000) Construction, analysis, ligation and self-assembly of DNA triple crossover complexes. J Am Chem Soc 122:1848-1860

22. Lagoudakis M, LaBean T (2000) 2-D DNA self-assembly for satisfiability. In: DNA based computers V. DIMACS, vol 54. American Mathematical Society, Providence, pp 141-154

23. Liu D, Wang M, Deng Z, Walulu R, Mao C (2004) Tensegrity: construction of rigid DNA triangles with flexible four-arm DNA junctions. J Am Chem Soc 126:2324-2325

24. Mao C, Sun W, Seeman NC (1999) Designed two-dimensional DNA holliday junction arrays visualized by atomic force microscopy. J Am Chem Soc 121:5437-5443

25. Park SH, Pistol C, Ahn SJ, Reif JH, Lebeck AR, Dwyer C, LaBean TH (2006) Finite-size, fully addressable DNA tile lattices formed by hierarchical assembly procedures. Angew Chem Int Ed 45:735-739 
26. Reif JH, Sahu S, Yin P (2005) Complexity of graph self-assembly in accretive systems and self-destructible systems. In: Lecture notes in computer science. Springer, Berlin, pp 257-274

27. Reif JH, Sahu S, Yin P (2006). Compact error-resilient computational DNA tilings. In: Nanotechnology: science and computation, pp 79-103

28. Revesz P (1990) Random walk in random and non-random environments. World Scientific, Singapore

29. Robinson R (1971) Undecidability and non periodicity of tilings of the plane. Invent Math 12:177-209

30. Rothemund P (2000) Using lateral capillary forces to compute by self-assembly. Proc Natl Acad Sci USA 97(3):984-989

31. Rothemund P (2001) Theory and experiments in algorithmic self-assembly. PhD thesis, University of Southern California

32. Rothemund P, Winfree E (2000) The program-size complexity of self-assembled squares (extended abstract). In: Proceedings of the thirty-second annual ACM symposium on theory of computing. ACM, New York, pp 459-468

33. Sa-Ardyen P, Jonoska N, Seeman NC (2003) Self-assembling DNA graphs. Nat Comput 2:427-438

34. Schulman R, Lee S, Papadakis N, Winfree E (2004) One dimensional boundaries for DNA tile self-assembly. In: DNA based computers 9. Lecture notes in computer science, vol 2943. Springer, Berlin, pp 108-125

35. Schulman R, Winfree E (2005) Programmable control of nucleation for algorithmic selfassembly. In: Lecture notes in computer science, vol 3384. Springer, Berlin, pp 319-328

36. Schulman R, Winfree E (2005) Self-replication and evolution of DNA crystals. In: The 13th European conference on artificial life (ECAL)

37. Soloveichik D, Winfree E (2006) Complexity of compact proofreading for self-assembled patterns. In: Lecture notes in computer science, vol 3892. Springer, Berlin, pp 305-324

38. Soloveichik D, Winfree E (2007) Complexity of self-assembled shapes. SIAM J Comput 36:1544-1569

39. Wang H (1961) Proving theorems by pattern recognition, II. Bell Syst Tech J 40:1-41

40. Winfree E (1998) Simulation of computing by self-assembly. Tech Rep 1998.22, Caltech

41. Winfree E (2006). Self-healing tile sets. In: Nanotechnology: science and computation, pp 5578

42. Winfree E, Bekbolatov R (2004) Proofreading tile sets: error correction for algorithmic selfassembly. In: DNA based computers 9. Lecture notes in computer science, vol 2943. Springer, Berlin, pp 126-144

43. Winfree E, Liu F, Wenzler LA, Seeman NC (1998) Design and self-assembly of twodimensional DNA crystals. Nature 394(6693):539-544

44. Winfree E, Yang X, Seeman NC (1999) Universal computation via self-assembly of DNA: some theory and experiments. In: Landweber L, Baum E (eds) DNA based computers II. DIMACS, vol 44. American Mathematical Society, Providence, pp 191-213

45. Yan H, LaBean TH, Feng L, Reif JH (2003) Directed nucleation assembly of DNA tile complexes for barcode-patterned lattices. Proc Natl Acad Sci USA 100(14):8103-8108

46. Yan H, Park SH, Finkelstein G, Reif JH, LaBean TH (2003) DNA-templated self-assembly of protein arrays and highly conductive nanowires. Science 301(5641):1882-1884 